\title{
Angular beaming and giant subpulses in the Crab pulsar
}

\author{
J. Gil ${ }^{1,2}$ and G. I. Melikidze ${ }^{1,3}$ \\ ${ }^{1}$ Institute of Astronomy, University of Zielona Góra, Lubuska 2, 65-265, Zielona Góra, Poland \\ e-mail: jag@astro.ia.uz.zgora.pl \\ 2 Department of Physics, University of Nevada Las Vegas, Las Vegas, NV 89154, USA \\ 3 Abastumani Astrophysical Observatory, Al. Kazbegi ave. 2a, Tbilisi 0160, Georgia
}

Received 13 December 2004 / Accepted 1 February 2005

\begin{abstract}
We propose that the extremely high brightness of the giant ultra-short subpulses observed in the Crab pulsar is consistent with the angular beaming due to relativistic motion of radiating sources along dipolar magnetic field lines. We argue that the effect of kinematic boosting inherently associated with localized relativistic sources results in apparent shortening and, in consequence, brightening of the emitted impulses. Thus we suggest that the giant subpulses are intrinsically many orders of magnitude less bright and powerful than they appear when interpreted within a temporal modulation model.
\end{abstract}

Key words. stars: pulsars: general: plasmas - pulsars: individual: the Crab pulsar

\section{Introduction}

Recently, a number of papers appeared claiming that the giant pulses represent the highest brightness temperatures $\left(\sim 10^{38} \mathrm{~K}\right)$ observed in the Universe (McLaughlin \& Cordes 2003; Cordes et al. 2004; Soglasnov et al. 2004). Even though only a few pulsars are known to emit such giant pulses (Lundgren et al. 1995; Cognard et al. 1996; Romani \& Johnston 2001; Johnston \& Romani 2003), understanding of their mechanism can potentially lead to understanding of a longstanding problem of the pulsar radio emission. The detection of extremely short and powerful (as compared with the mean flux of about $10 \mathrm{mJy}$ ), 2-nanosecond - $1000 \mathrm{Jy}$, subpulses within the radio giant pulses from the Crab pulsar has been reported by Hankins et al. (2003, HKWE03 hereafter). HKWE03 argued that these nanosecond giant subpulses were true temporal modulations associated with an explosive collapse of nonlinear plasma turbulence (Weatherall 1998). The necessary energy density was estimated by HKWE03 as $E_{\mathrm{r}} \sim 2 \times 10^{14} \mathrm{erg} \mathrm{cm}^{-3}$. The maximum available (spin-down) kinetic energy density is $E_{\mathrm{k}}=$ $n_{\mathrm{GJ}} \gamma m c^{2} \sim 10^{17}(R / r)^{3} \mathrm{erg} \mathrm{cm}^{-3}$, where $n_{\mathrm{GJ}}$ is the GoldreichJulian (1969) number density, $\gamma$ is the Lorentz factor of particles accelerated by full polar gap potential drop (Ruderman \& Sutherland 1975), $R=10^{6} \mathrm{~cm}$ is the neutron star radius and $r$ is the altitude (radius) of the emission region. As one can see, in the radio emission region with expected altitudes $r / R \gtrsim 10$ (e.g. Kijak \& Gil 1998) $E_{\mathrm{r}}$ exceeds the entire available spin-down energy density. Thus, a temporal modulation model seems to have a serious energetic problem, unless giant pulses originate much closer to the polar cap surface. In this letter we argue that both the time-scale and the energetics of the Crab pulsar giant nanosecond subpulses are consistent with the angular beaming due to the curvature of dipolar field lines (different from the conventional angular beaming due to the pulsar rotation), thus they are consistent with the coherent curvature radiation (e.g. Melikidze et al. 2000; Gil et al. 2004). We therefore propose an alternative explanation for the nano-giant subpulses to that of the temporal modulation model, which invokes steady radiation process that is glimpsed only for 2 nanoseconds. This angular beaming model does not require such an extreme values of luminosity and brightness temperature.

\section{The time-scales}

\subsection{The radiation time-scale}

Let us consider a stream of localized sources moving relativistically between points A and B in Fig. 1a and assume that some of them are distinguished by emitting slightly more power $\mathcal{P}$ than the average. From the observational point of view it means that there are very few sources emitting towards the observer during the alignment time. For simplicity let us first consider a single source, which is marked in Fig. 1a in three different positions A, B and C. Following Jackson (1975) we can argue that the duration of the observed impulse related to the curvature of field lines is much shorter than the duration predicted by Eq. (4) below. Let us consider two particular points along a given dipolar field line: first (A) at which the source $S_{1}$ becomes aligned (ray 1) and second (B) at which it becomes misaligned (ray 2) with the observer $O$ (Fig. 1a). The source covers a distance $l=\rho / \gamma$ between these points during a time interval $\delta t=\rho \gamma^{-1} / v$. During this time the radiation emitted at the first alignment point (A) travels a distance $L=c \delta t=(c / v) \rho \gamma^{-1}=\rho /(\beta \gamma)$. Thus, the radiation overtakes 
the source only by a distance $\Delta=L-l=(1 / \beta-1)(\rho / \gamma) \approx \rho / \gamma^{3}$ (when the radiation emitted at point $\mathrm{A}$ reaches point $\mathrm{B}$, the source of this radiation arrives at point $\mathrm{C}$ ). This is the pulse length in space, and thus the duration of the observed impulse emitted by the considered source towards an observer is

$\Delta \tau=\frac{\Delta}{c}=\frac{\rho}{c \gamma^{3}}=\gamma^{-2} \Delta t_{\mathrm{rad}}$,

(Jackson 1975; Rybicki \& Lightman 1979) where $\Delta t_{\text {rad }}$ is the radiation time-scale (that is an intrinsic duration of the continuous or temporal radiation). If the radiation process is longer than the duration of visibility of its source(s), then the alignment time-scale $\Delta t_{\mathrm{al}}$ (see Eq. (1) below) should appear in the right hand side of Eq. (1) instead of $\Delta t_{\mathrm{rad}}$. Therefore, if the source longitudinal (along field lines) dimension is smaller than $\Delta \approx \rho / \gamma^{3}$, then the apparent duration of the impulse $\Delta \tau$ is $\gamma^{2}$ times shorter than the actual time of radiation emitted towards an observer. It is important to emphasize that both $\Delta t$ and $\Delta \tau$ refer to the same observer's frame of reference. This effect occurs for any localized source of radiation moving towards the observer with a speed slightly lower than that of the emitted waves. More precisely, the center of mass of the source should move towards an observer (relativistic bulk motion) e.g. Ginzburg (1979), Zhang \& Meszaros (2004). Gil (1985) suggested that this effect can be applied to the duration of the shortest microstructure in the pulsar radiation, down to nanosecond time scales. The observed emission would then result from an incoherent overlapping of a large number of such nanosecond shots, consistent with the amplitude modulated shot noise model (Cordes 1976; Gil \& Snakowski 1990).

Both isolated 2-ns giant subpulses, as well as a sequence of a number of such subpulses (when unresolved this sequence constitutes a normal giant pulse in our view), can be seen in Fig. 1 of HKWE03. Within our model such case corresponds to a sequence of sources, each having a longitudinal dimension smaller than $\Delta$ and separation between the adjacent sources larger than $\Delta$, where $\Delta \approx \rho / \gamma^{3}$ is the spatial length of the impulse associated with each source.

\subsection{The alignment time-scale}

Let us consider a localized (point-like) source of the broadband coherent curvature radiation, moving relativistically (with the Lorentz factor $\gamma=\left(1-\beta^{2}\right)^{-1 / 2}$, where $\left.\beta=v / c \sim 1\right)$ along a narrow bundle of dipolar magnetic field lines with a radius of curvature

$\rho=9.2 \times 10^{7} r_{6}^{1 / 2} P^{1 / 2} s^{-1} \mathrm{~cm}$,

where $s=d / r_{\mathrm{p}}$ is the normalized polar coordinate of the central line of the bundle, $r_{6}=r / R$ is the normalized emission altitude $r$ (for the frequency $v=5 \mathrm{GHz}, r_{6} \sim 10$ in the Crab pulsar, Kijak \& Gil 1998), $d$ is the distance from the dipolar axis to the foot of a dipolar field line, $r_{\mathrm{p}} \approx 10^{4} P^{-1 / 2}$ is the polar cap radius, $R=10^{6} \mathrm{~cm}$ is the neutron star radius and $P$ is the pulsar period. The geometry is schematically illustrated in Fig. 1b, where a narrow bundle of field lines is marked. The curvature radiation is emitted along dipolar field lines into a narrow cone

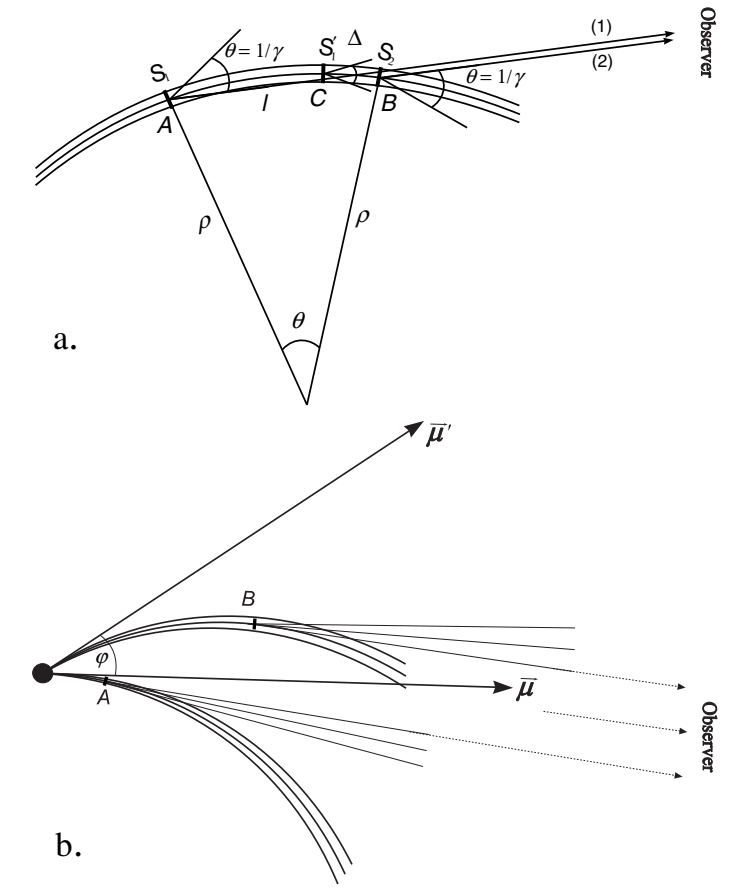

Fig. 1. a) Geometry of the observed curvature radiation emitted by the source $(S)$ moving relativistically along a narrow bundle of dipolar field lines with the radius of curvature $\rho$. b) Schematic representation of the flux tube rotating with the star. Two different orientations of the magnetic axes are marked $\boldsymbol{\mu}$ (earlier) and $\boldsymbol{\mu}^{\prime}$ (later). See text for explanation of other symbols.

with an opening angle $\theta=1 / \gamma($ e.g. Jackson 1975). For a fixed arrangement of the observer's direction, the field line polar coordinate $s$ and the frequency dependent emission altitude $r_{6}(v)$, the source is aligned within $1 / \gamma$ with the observer during the time interval $\delta t=l / v=\rho \theta / v=\rho \gamma^{-1} / v$ (see Fig. 1b). Here $\rho$ is described by Eq. (2), provided that $\delta t$ is longer than rotational time-scale

$\Delta t_{\mathrm{rot}}=\gamma^{-1} \frac{P}{2 \pi}$,

corresponding to the conventional angular beaming. Therefore, we can define an "alignment time"

$\Delta t_{\mathrm{al}}=\frac{\rho}{\gamma c}$

during which the observer is aligned with the relativistic source (emitting into a narrow $1 / \gamma$ cone) by means of the geometrical optics. It is worth noting that the alignment time-scale $\Delta t_{\mathrm{al}}$ exceeds that of the rotational time-scale $\Delta t_{\text {rot }}$ if the radius of the curvature of field lines (Eq. (2)) $\rho>c P / 2 \pi$. Thus, in the Crab pulsar this means that $\rho>1.6 \times 10^{8} \mathrm{~cm}$. HKWE03 argued that the conventional angular beaming (Eq. (3)) cannot explain the 2-nanosecond duration of their giant subpulses, since it requires $\gamma \sim 10^{6}$, much above the expected values $\gamma<1000$. Therefore, they concluded that their giant subpulses represent true temporal modulations of emitting regions. However, as we argue below these ultra-short time scales are perfectly consistent with another kind of angular beaming due to relativistic motion of localized sources of coherent radio emission along 
dipolar magnetic field lines, like in the case of curvature radiation. Therefore, we believe that there is no need to invoke such rapid modulations of emitting regions, at least from the point of view of the observed time-scales.

\subsection{True alignment time-scale}

Let us estimate the time-scales we introduced in the previous subsections for the Crab pulsar $(P=0.03352 \mathrm{~s})$. The alignment time-scale (Eq. (4)) can be estimated as $\Delta t_{\mathrm{al}}=3.3 \times 10^{-5} \rho_{8} \gamma_{2}^{-1}$. Here $\rho_{8}=\rho \times 10^{-8}$ and $\gamma_{2}=\gamma \times 10^{-2}$ are the normalized curvature radius and Lorentz factor, respectively. Then we can estimate the rotation time-scale as $\Delta t_{\text {rot }}=5.3 \times 10^{-5} \gamma_{2}^{-1}$ (Eq. (3)) and the radiation time-scale as $\Delta t_{\mathrm{rad}}=2 \times 10^{-5} \gamma_{2}^{2}$ (Eq. (1)). In order to observe the maximal pulse shortening effect we need that all these time-scales to be of the same order $\Delta t_{\mathrm{al}} \sim$ $\Delta t_{\text {rot }} \sim \Delta t_{\text {rad }}$ (see Fig. 1b). This condition can be satisfied only for rather small $\gamma_{2} \sim 1$. On the other hand the suggested scenario implies that the longitudinal size of the source of the giant subpulses is about $d_{\|}=60 \mathrm{~cm}$. Therefore, the source should exist (without dispersing along magnetic field lines) during the survival time-scale $\Delta t_{\mathrm{sr}}=2 \gamma^{2} d_{\|} / c \sim 4 \times 10^{-5} \gamma_{2}^{2}$ s. Let us assume that the slab (with dimensions $d_{\|}$and $d_{\perp}$ ) of the turbulent relativistic plasma, which is the source of the giant subpulses, is formed in the magnetosphere due to sparking process on the polar cap (e.g. Asseo \& Melikidze 1998). The slab must survive during the time necessary to reach the emission altitude, where the two-stream instability develops and initiates a radiation process. Then we can write the condition $\Delta t_{\mathrm{sr}} \sim r / c \sim 3.3 \times 10^{-5} r_{6}$ or $4 \times 10^{-5} \gamma_{2}^{2} \sim 3.3 \times 10^{-4}$. Consequently we can conclude that the particles of the slab should posses the Lorentz factors about $\gamma_{2} \sim 3$ in order to reach the emission altitude ${ }^{1}$ and still be localized within $d_{\|}$.

However with $\gamma_{2} \sim 3$, neither the alignment time-scale Eq. (4) nor the rotational time-scale is long enough to correspond to the radiation time $\Delta t_{\text {rad }} \sim \gamma^{2} \Delta \tau_{\text {obs }}$ following from Eq. (1). As we will argue below the combination of both relativistic flow along dipolar field lines and the pulsar rotation can give under some condition the right time-scales for the radiation processes. This is illustrated in Fig. 1b, where the moving slab-like source in two different positions along the same bundle of the field lines is marked by $A$ and $B$, respectively. Let us assume that at time $t=0$ the magnetic axis of our orthogonal rotator is aligned with an observer, defining on a reference observing angle $\phi=0$. Due to the pulsar rotation with the angular velocity $\Omega=2 \pi / P$, the angle between $\mu$ and the reference direction (to the observer) varies as $\phi=\Omega t$. Let us now consider a flux tube of dipolar field lines encompassed by a slab-like source of length $d_{\|} \sim 60 \mathrm{~cm}$ and the cross-section $S_{0}$. As the

\footnotetext{
1 After Kijak \& Gil (1998) we have assumed that $r_{6} \sim 10$, but in the short period Crab pulsar the radius of curvature of magnetic field lines is appropriate for the curvature radiation even at altitudes close to the light cylinder. In fact, if the radiation comes from higher up, then the radius of curvature and hence the alignment time-scale (Eq. (4)) is longer, requiring even smaller intrinsic luminosity. However, such a radiation should not be considered as the outer gap emission, since by means of physical mechanism it is just a polar cap related radiation originating near the light cylinder.
}

radiating slab flows along dipolar field lines and rotates with the pulsar, its radiation becomes aligned with the observer during the time $\Delta \sim 10^{-4} \mathrm{~s}$. This can be compared with the much shorter alignment time $\Delta t_{\mathrm{al}} \sim 5 \times 10^{-5}$ (for $s_{0} \sim 0.1$ ) when the pulsar rotation is ignored. But even more important conclusion is that lengthening of the true alignment time works only for the trailing side of the pulse profile centered on the fiducial phase determined by the projection of the magnetic axis on the sky. Therefore, we can predict that the strongest and the narrowest giant subpulses should lag the peak of the profile, both in the main pulse and in the interpulse. Although the exact location of the giant subpulses for the Crab pulsar is not known, such an effect is clearly visible in the millisecond pulsar B1937+21 (see Fig. 2 in Cognard et al. 1996).

\section{Energetics}

As reported by HKWE03, the ultra-short nano-giant subpulses detected at $v=5 \mathrm{GHz}$ in the Crab pulsar often exceed fluxes $S=1000 \mathrm{Jy}=10^{-20} \mathrm{erg} \mathrm{s}^{-1} \mathrm{~Hz}^{-1} \mathrm{~cm}^{-1}$. These fluxes can be converted into the brightness temperature $T_{\mathrm{b}}=$ $S D^{2} /\left[2 k(v W)^{2}\right]$, where $D=2 \mathrm{kpc}=6 \times 10^{21} \mathrm{~cm}$ is the distance to the pulsar, $k$ is the Boltzmann constant and $W$ is the time scale of the corresponding emission process (e.g. McLaughlin $\&$ Cordes 2003). Assuming that $W=2 \times 10^{-9}$ s (HKWE03) one obtains the extraordinarily high brightness temperatures $T_{\mathrm{b}} \sim 10^{38} \mathrm{~K}$, implying by far the most luminous emission from any astronomical object. However, within our model the actual emission process corresponding to a giant subpulse of duration $\Delta \tau=2 \times 10^{-9}$ s occurs over a much longer "alignment" time interval $W=\Delta t=\gamma^{2} \Delta \tau$ (Eqs. (4) and (1)), which leads to $\gamma^{4}$ times lower brightness temperatures. Since $\gamma$ is of the order of 100 , then $T_{\mathrm{b}}<10^{30} \mathrm{~K}$, consistent with normal giant pulses in the Crab pulsar and other pulsars (see Fig. 1 in McLaughlin \& Cordes 2003).

Let us then consider a localized source(s) moving along a dipolar field line between points $\mathrm{A}$ and $\mathrm{B}$ and emitting towards the observer $O$ steady coherent radiation with an intrinsic power $\mathcal{P}$ (Fig. 1a). Because of the curved trajectory, a distant observer will receive pulse of radiation associated with each source. While moving over the alignment distance $l=\rho \gamma^{-1}$, the energy emitted towards $O$ is $E=\mathcal{P} \Delta t$, where $\Delta t=\rho \gamma^{-1} / c$ (Eq. (4)) is the alignment time interval. This energy will be received by the observer $O$ in a much shorter time $\Delta \tau=\gamma^{-2} \Delta t$ (Eq. (1)), and therefore the apparent luminosity

$\mathcal{L}=\frac{E}{\Delta \tau}=\gamma^{2} \mathcal{P}$

Thus, the nanosecond giant subpulses appear to be $\gamma^{2}=\gamma_{2}^{2} \times$ $10^{4}$ times more luminous than the intrinsic power of their source(s). Besides the apparent fluxes $S \propto \mathcal{L}$ are also $\gamma^{2}$ times overestimated. It is important to emphasize that this kinematical boosting is a direct consequence of apparent shortening of the impulse duration described by Eq. (1).

The apparent fluxes $S \sim 1000 \mathrm{Jy}$ of the nanosecond giant subpulses can be formally converted into the emitted luminosity $\mathcal{L}=S \theta^{2} D^{2} \Delta v$. With $D=2 \mathrm{kpc}, \theta=\gamma_{2}^{-1} \times 10^{-2}$ and $\Delta v=5 \times 10^{8} \mathrm{~Hz}$ one obtains $\mathcal{L} \sim \gamma_{2}^{-2} \times 10^{28} \mathrm{erg} / \mathrm{s}$. This is 
an extremely large value, comparable with the total radio luminosity of the Crab pulsar (Taylor et al. 1993). Of course, this argument is not independent from the extraordinarily high brightness temperatures $T_{\mathrm{b}} \sim 10^{38} \mathrm{~K}$ derived in Sect. 3.1). HKWE03 suggested that they have detected such extremely powerful and bright sources. We propose an alternative explanation which does not require such extreme energetic conditions. In fact, according to Eq. (5), the actual power of the emitted radiation $\mathcal{P}=\gamma^{-2} S \theta^{2} D^{2} \Delta v=\gamma^{-2} \mathcal{L}$, which for the parameters given above yields approximately

$\mathcal{P} \sim \gamma_{2}^{-4} \times 10^{24} \mathrm{erg} \mathrm{s}^{-1}$.

This is still a very large power (consistent with $T_{\mathrm{b}} \lesssim 10^{30} \mathrm{~K}$ ) but much smaller than the total radio luminosity of the Crab pulsar.

\section{Discussion}

The 2-nanosecond, 1-kJy bursts of radiation observed by HKWE03 imply enormous instantaneous luminosity of about $10^{28} \mathrm{erg} / \mathrm{s}$, comparable with the total radio luminosity of the Crab pulsar, or equivalently, the highest ever inferred brightness temperature of about $10^{38} \mathrm{~K}$. The above estimates are correct under the assumption that the actual emission process is as short as 2 nanoseconds. HKWE03 argued that this extremely high power was due to the short-lived (2 nanoseconds) release of energy stored in the collapsing wave packet (Weatherall 1998). In this letter we proposed an alternative model, which invokes steady radiation process that is glimpsed only for a short interval ( $2 \mathrm{~ns})$. We argued that the short observed time scale results from angular beaming due to the relativistic motion of localized sources along dipolar field lines, which involves a well known "kinematic boosting" effect that shortens an apparent duration and consequently increases an apparent brightness of the observed signal. One can argue that each localized source has to emit only about $10^{21} \mathrm{erg} / \mathrm{s}$ and the actual brightness temperature does not exceed $10^{30} \mathrm{~K}$. Our model can be applied to any impulsive radiation whose source is approaching an observer in relativistic motion, perhaps even to the collapsing wave impulse of HKWE03. However, the coherent curvature radiation is the most natural emission mechanism to consider, and we will demonstrate that this kind of radiation can really satisfy the observational constraints in a separate paper. Here we only want to emphasize that the condition $\lambda<\Delta=\rho / \gamma^{3}=\left(\rho_{8} / \gamma_{2}^{3}\right) \times 10^{2}$ is naturally satisfied at radio wavelengths, where $\lambda$ is the longitudinal source dimension. Note that $\Delta=60 \mathrm{~cm}$ (which of course corresponds to 2 light nanoseconds).

Generally, within our model the following two steps are involved: in the first step the localized source(s) should be somehow distinguished from the background radiation. Then, in the second step, the radiation intensity of distinguished source(s) can be kinematically boosted by means of Eq. (5). It seems that such distinguished source should be associated with exceptionally intense sparks on the polar cap, which can deliver more dense and/or more energetic plasma stream(s). Interestingly, pulses exhibiting giant pulses are distinguished by highest values of the magnetic field $B_{\mathrm{LC}} \gtrsim 10^{5} \mathrm{G}$ at the light cylinder,
Table 1. Giant pulse candidates.

\begin{tabular}{cccccc}
\hline \hline PSR J & $\begin{array}{c}P \\
(\mathrm{msec})\end{array}$ & $\begin{array}{c}\dot{P} \\
\left(10^{-15}\right)\end{array}$ & \multicolumn{2}{c}{$\begin{array}{c}B_{\mathrm{LC}} \\
\left(10^{5} \mathrm{G}\right)\end{array}$} \\
\hline $\mathbf{0 5 3 4 + 2 2 0 0}$ & 33.1 & 423 & 252 & 9.7 & $(2)$ \\
$\mathbf{0 5 4 0}-\mathbf{6 9 1 9}$ & 50.4 & 479 & 199 & 3.6 & $(5)$ \\
$1513-5908$ & 151 & 1540 & 137 & 0.42 & $(31)$ \\
$1124-5916$ & 135 & 747 & 120 & 0.38 & $(32)$ \\
$1617-5055$ & 69.4 & 135 & 113 & 0.86 & $(16)$ \\
$1420-6048$ & 68.2 & 83.2 & 99 & 0.70 & $(21)$ \\
$1119-6127$ & 408 & 4020 & 95 & 0.06 & $(108)$ \\
$\mathbf{0 8 3 5}-\mathbf{4 5 1 0}$ & 89.3 & 125 & 94 & 0.45 & $(30)$ \\
$\mathbf{1 8 2 4}-\mathbf{2 4 5 2}$ & 3.05 & 0.0016 & 33 & 7.3 & $(3)$ \\
$1823-30 \mathrm{~A}$ & 5.44 & 0.0034 & 28 & 2.5 & $(7)$ \\
$\mathbf{1 9 3 9}+\mathbf{2 1 3 4}$ & 1.56 & 0.0001 & 23 & 10 & $(1)$ \\
$\mathbf{0 2 1 8}+\mathbf{4 2 3 2}$ & 2.32 & 0.00008 & 17 & 3.2 & $(6)$ \\
$\mathbf{1 9 5 9}+\mathbf{2 0 4 8}$ & 1.61 & 0.00002 & 14 & 3.7 & $(4)$ \\
$2129+1210 \mathrm{E}$ & 4.65 & 0.0002 & 13 & 0.84 & $(19)$ \\
\hline
\end{tabular}

suggesting an outer gap emission mechanism. However, this can also point towards the inner gap related emission. In fact, one can show that $B_{\mathrm{LC}} \sim a^{2} / P$, where $P$ is the pulsar period, $a=r_{\mathrm{p}} / h$ is the complexity parameter of the polar cap (Gil \& Sendyk 2000), $r_{\mathrm{p}} \sim 10^{4} P^{-1 / 2}$ is the polar cap radius and $h$ is the actual height of the inner gap. Since the potential drop across the gap $\Delta V \propto h^{2}$ and $h<r_{\mathrm{p}} / \sqrt{2}$ (Ruderman \& Sutherland $1975)$, then the quantity $a^{2}=\Delta V_{\max } / \Delta V$ describes the reservoir of the maximum available potential drop across the polar cap (over the actual value determined by $h$ ), which can be occasionally used to create exceptionally energetic spark(s)/plasma stream(s). Such events can occur when the actual gap height $h$ becomes a significant fraction of the polar cap radius $r_{\mathrm{p}}$.

The values of the complexity parameter $a$ can well exceed 100 in normal pulsars, while in millisecond pulsars they are limited to about 40 (see Fig. 1 in Gil \& Sendyk 2000). Therefore, when pulsars in both groups are ranked with respect to the parameter $a$, pulsars with giant pulses occupy the top of the lists (Table 1). The Vela pulsar, which was reported to exhibit some kind of giant pulse behavior (Johnston et al. 2001) is also very high on the list. Moreover, sporadic large amplitude pulses (LAP) from two millisecond pulsars (J1959+2048 and $\mathrm{J} 0218+4232$ ) have been reported quite recently by Joshi et al. (2004). Although $B_{\mathrm{LC}}$ is a good parameter to make a list of giant pulse candidates, only a value of $a$ has a physical meaning within our model. In Table 1 we propose a number of candidates for giant pulses with high values of the complexity parameter ( $a>10$ for millisecond pulsars and $a>90$ for normal pulsars) and relatively low values of $B_{\mathrm{LC}}$. Pulsars marked in boldface show giant pulses. Numbers in parenthesis denote ranking position in $B_{\mathrm{LC}}=9.3 \dot{P}^{0.5} P^{-2.5}$. The complexity parameter $a=5 \dot{P}^{0.29} P^{-0.64}$. PSR J1119-6127 with low $B_{\mathrm{LC}} \approx 6 \times 10^{3} \mathrm{G}$ and high $a=95$ seems to be the most interesting case. 
Thus, according to our scenario, both ordinary and giant pulses are related to the inner gap sparking activity and originate at relatively low altitudes. This is contrary to the suggestion that the latter arise in the outer gap region (Romani \& Johnston 2001), which is motivated by the observational fact that giant pulses and high energy emission (X-rays, gammarays) seem to occur at approximately the same ranges of longitudes. If the high energy emission originates within outer gaps, then giant pulses should also be associated with these outer regions. However, there are models of high energy emission originating close to the polar caps (for review see Baring 2004), and our model of giant subpulses in the Crab pulsar appeals to this class of models.

Finally, the emission statistics should be shortly discussed. It is well known that the amplitude distribution of giant pulses appears to follow a power-law rather than the log-normal distribution seen in normal pulses. If the giant pulses to some extent (or even completely) correspond to the elementary non-linear processes such as turbulence, e.g. solitons produced via modulational instability (Melikidze et al. 2000; Gil et al. 2004), then they should exhibit a power-law in their cumulative probability distribution (Cairns et al. 2003). The normal radio emission must represent an incoherent addition of very large number of independent coherent shots. As a result, the evidence for elementary non-linearity will be lost in the linear process of ensemble averaging, which should result in the log-normal distribution (Cairns et al. 2003).

Acknowledgements. This paper is supported in part by the Grant 1 P03D 02926 of the Polish State Committee for Scientific Research. J.G. acknowledges support of NASA grant NNG04GD51G during his stay in Las Vegas and thanks Bing Zhang and Jarek Dyks for helpful discussions. We thank E. Gil and M. Margishvili for technical help.

\section{References}

Asseo, E., \& Melikidze, G. I. 1998, MNRAS, 301, 59

Baring, M. 2004, AdSpR, 33, 552

Cairns, I. H., Johnston, S., \& Das, P. 2003, MNRAS, 343, 512

Cordes, J. M., Bhat, N. D., Hankins, T. H., et al. 2004, ApJ, 612, 375

Cognard, L., Shrauner, J. A., Taylor, J. H., et al. 1996, ApJ, 457, L81

Cordes, J. M. 1976, ApJ, 210, 780

Gil, J. 1985, Ap\&SS, 110, 293

Gil, J., Lyubarsky, Y., \& Melikidze, G. I. 2004, ApJ, 600, 872

Gil, J., \& Snakowski, K. 1990, A\&A, 234, 269

Gil, J., \& Sendyk, M. 2000, ApJ, 541, 351

Ginzburg, V. L. 1979, Theoretical physics and Astrophysics, Sect. 5 (Oxford: Pergamon Press)

Goldreich, P., \& Julian, W. 1969, ApJ, 157, 869

Hankins, T. H., Kern, J. S., Weatherall, J. C., et al. 2003, Nature, 422, 141 (HKWE03)

Jackson, J. D. 1975, Classical electrodynamics (New York: John Wiley \& Sons)

Johnston, S., van Straten, W., Kramer, M., Bailes, et al. 2001, ApJ, 549, L101

Johnston, S., \& Romani, R. W. 2003, ApJ, 590, L95

Joshi, B. C., Kramer, M., Lyne, A. G., et al. 2004, Proc. IAU Symp. 218, ed. F. Camilo, \& B. Gaensler, 319

Kijak, J., \& Gil, J. 1998, MNRAS, 299, 855

Lundgren, S. C., Cordes, J. M., Ulmer, M., et al. 1995, ApJ, 453, 433

McLaughlin, M. A., \& Cordes, J. M. 2003, ApJ, 468, 779

Melikidze, G. I., Gil, J., \& Pataraya, A. D. 2000, ApJ, 544, 1081

Rickett, B. 1975, ApJ, 197, 185

Romani, R. W., \& Johnston, S. 2001, ApJ, 557, L93

Ruderman, M., \& Sutherland, P. G. 1975, ApJ, 196, 51

Rybicki, G., \& Lightman, A. 1979, Radiative Processes in Astrophysics (New York: Wiley)

Soglasnov, V. A., Popov, M. V., Bartel, N., et al. 2004, ApJ, 616, 439

Sturrock, P. A. 1971, ApJ, 164, 529

Taylor, J. H., Manchester, R. N., \& Lyne, A. G. 1993, ApJS, 88, 259

Weatherall, J. C. 1998, ApJ, 506, 341

Zhang, B., \& Meszaros, P. 2004, Int. J. Mod. Phys. A, 19 (15), 2385 